Original Article

\title{
Reliability analysis of the Korean version of the trunk control measurement scale in cerebral palsy
}

\author{
Ju-Young Heo, PT, MSc ${ }^{1)}$, Hwa-Kyung Shin, PT, PhD²)* \\ 1) Department of Physical Therapy, The Graduate School, Daegu Catholic University, \\ Republic of Korea \\ 2) Department of Physical Therapy, College of Bio and Medical Science, Daegu Catholic University: \\ 13-13 Hayang-Ro, Hayang-Eup, Gyeongsan-si, Gyeongbuk 38430, Republic of Korea
}

\begin{abstract}
Purpose] The purpose of this study was to translate the Trunk Control Measurement Scale into a Korean version and to analyze the intra- and inter-rater reliability. [Subjects and Methods] Fifteen children with spastic cerebral palsy and four physical therapists with over 10 years of clinical experience participated in this study. A Korean-American physical therapist translated the trunk control measurement scale from English into a Korean version. Four physical therapists viewed the video data of 15 children and scored each child's trunk control measurement scale performance on seven separate days. Four testers analyzed the test-retest reliability and inter-rater reliability using the intra-class correlation coefficient. [Results] Intra-class correlation coefficients for test-retest and inter-rater reliability for the Korean trunk control measurement scale showed significantly high reliability in all testers and sublevels. [Conclusion] The Korean version of the measurement scale is a reliable and suitable instrument for assessing trunk control in individuals with cerebral palsy in Korea.

Key words: Cerebral palsy, Reliability, Trunk control measurement scale
\end{abstract}

(This article was submitted Jun. 8, 2017, and was accepted Oct. 4, 2017)

\section{INTRODUCTION}

Cerebral palsy $(\mathrm{CP})$ refers to permanent damage to an individual's movements and postural development ${ }^{1)}$. In particular, the postural control of deficient trunk muscles is subject to the greatest damage in patients with $\mathrm{CP}^{2)}$. Postural controls involve the coordination of the musculoskeletal system to satisfy an action objective of orientation and stability, which is achieved through the interaction of the somatic sensory and motor systems ${ }^{3}$. Trunk control disorders can directly affect activities of daily living, such as sitting, stretching the arms, or walking ${ }^{4}$. Nonetheless, few studies have been conducted on trunk control in children with $\mathrm{CP}$.

One important priority is to establish guidelines for the measurement tools and therapeutic interventions for trunk control. The Seated Postural Control Measure (SPCM) or the Spinal Alignment and Range of Motion Measure (SAROMM) have been used to evaluate trunk control in clinical practice ${ }^{5,6)}$. However, these tools only evaluate static sitting, even though dynamic sitting is more related to functional activity.

The Trunk Control Measurement Scale (TCMS) was developed by Belgian and German clinical researchers in 2011 7,8 , and it is based on the Trunk Impairment Scale (TIS). It measures all aspects of the static and dynamic states of children with $\mathrm{CP}$ and assesses the essential elements of trunk control based on functional activities. It includes static sitting balance ( 5 items) and dynamic sitting balance tests, and the dynamic sitting balance testing is subdivided into selective movement control (7 items) and dynamic reaching tests (3 item). This can be measured on both sides, and the total score ranges from

*Corresponding author. Hwa-Kyung Shin (E-mail: hkshin1@cu.ac.kr)

(C2018 The Society of Physical Therapy Science. Published by IPEC Inc. 
Table 1. Test-retest reliability on sublevel of K-TCMS

\begin{tabular}{lclcc}
\hline \multicolumn{1}{c}{ Sublevel } & \multicolumn{1}{c}{ Tester 1 } & \multicolumn{1}{c}{ Tester 2 } & \multicolumn{1}{c}{ Tester 3 } & \multicolumn{1}{c}{ Tester 4 } \\
\hline Static sitting balance & $0.972^{\mathrm{a}}(0.916-0.991)^{\mathrm{b},{ }^{*}}$ & $0.999(0.998-1.000)$ & $0.998(0.994-0.999)^{*}$ & $0.998(0.995-0.999)^{*}$ \\
Dynamic sitting balance & $0.979(0.936-0.993)^{*}$ & $0.999(0.998-1.000)^{*}$ & $0.995(0.984-0.998)^{*}$ & $0.986(0.960-0.995)^{*}$ \\
Dynamic reaching & $0.941(0.826-0.980)^{*}$ & $1.000^{*}$ & $0.996(0.987-0.998)^{*}$ & $0.997(0.991-0.999)^{*}$ \\
\hline
\end{tabular}

${ }^{\mathrm{a}} \mathrm{ICC}$ : Intraclass correlation coefficient, ${ }^{\mathrm{b}} \mathrm{CI}$ : Confidence interval.

Value are Mean \pm Standard Deviation. ${ }^{*}<0.05$.

0 to 58 points. The higher the score, the better the function. The intraclass correlation coefficient (ICC) for the intra-rater reliability of the TCMS was 0.98 , and the ICC for test-retest reliability was 0.97 , which showed a high reliability ${ }^{7,8)}$. Despite the high validity and reliability of the original TCMS, it has not been widely used in Korea until now. Thus, we aimed to translate the TCMS into Korean and to validate the reliability of the Korean version of the TCMS (K-TCMS) as part of a foundational study with the goal of standardization.

\section{SUBJECTS AND METHODS}

A total of 15 children with CP (7 males, 8 females) participated in our study. Selection criteria included the following: subjects in stages I, II, and III according to the gross motor function classification system (GMFCS) who could walk independently, move using assistive tools, and who had not had any orthopedic surgery or intervention or taken any medication within the last 6 months. All subjects were fully informed of the test procedure in advance, and their participation in the test was voluntary. The mean age of the subjects was $8.13 \pm 2.61$ years. Hemiplegia, diplegia, and triplegia were present in 3 , 10, and 2 patients, respectively. In terms of the GMFCS staging, 4, 6, and 5 patients were stage I, II, and III, respectively. This study was approved by the ethical committee of the Faculty of Physical Therapy at the Catholic University of Daegu (CUIRB-2016-0009).

This study was first authorized as a Korean localization of the TCMS by Lieve Heyrman, the original author of the TCMS, and a translation verification committee translated the TCMS and modified it after vigorous discussion ${ }^{9}$. Discrepancies between the Korean and original versions or difficulties in interpretation or application were resolved through an exchange of communications and discussions with experts, as well as advice from the original author, thereby enabling the K-TCMS to be adapted to the Korean culture and language. Then, the K-TCMS was again translated into English (back translation) to re-authorize the contents of the original and to verify the validation of the interpretation.

Video shooting was conducted while children performed the various assessment tests in the TCMS. Children wore clothes that allowed their trunk to be observed during the test, and assistive tools and shoes were not worn while performing the assessments. Children completed the tests according to the assessor's instructions. To help the subjects understand the tests, the assessors gave demonstrations that the children were instructed to follow. If the children had any difficulty understanding, an assessor assisted the children with their hands to direct the motion correctly. Children were recommended to repeat the motion up to 3 times.

Four therapists who did not participate in the video shooting or the assessments scored the children while watching the recorded videos independently to perform the reliability validation. The assessors that were selected had clinical treatment experience in pediatric physical therapy with specialization in central nervous system development for 8 years ( 2 assessors) and 10 years ( 2 assessors). Each of the assessors was fully informed of the guidelines prior to the assessment. During the first assessment, 15 children were assessed randomly; after 7 days, the second assessment was conducted. Then, after 3 days, 5 children were chosen randomly for another assessment.

The statistical analysis in this study was done using SPSS 24.0. Intraclass correlation coefficients (ICCs) were used to determine the test-retest reliability and inter-rater reliability of the K-TCMS. Statistical significance was set at $\mathrm{p}<0.05$.

\section{RESULTS}

ICCs for the test-retest reliability of K-TCMS showed significantly high reliability in all testers and in all sublevels $(\mathrm{p}<0.05)$ (Table 1). ICCs for the inter-rater reliability of K-TCMS showed significantly high reliability between 4 testers and in all sublevels $(\mathrm{p}<0.05)$ (Table 2$)$.

\section{DISCUSSION}

This study created the K-TCMS after a verification process of translation and back translation in order to promote the implementation of the TCMS in Korea. To evaluate the reliability of the K-TCMS, 15 children with CP were chosen as subjects and assessed independently by 4 assessors to validate the test-retest and inter-rater reliabilities.

The objective of the localization of the assessment tool was to avoid a literal translation and to reduce cultural differences by using words and terms that are relevant to the Korean culture ${ }^{10)}$. In previous papers related to other assessment tools, back 
Table 2. Inter-rater reliability on sublevel of K-TCMS

\begin{tabular}{|c|c|c|c|c|}
\hline TCMS & mean $\pm \mathrm{SD}$ & $\mathrm{ICC}^{\mathrm{a}}$ & $95 \% \mathrm{CI}^{\mathrm{b}}$ & $\mathrm{p}$ \\
\hline \multicolumn{5}{|c|}{ Static sitting balance } \\
\hline Tester 1 & $16.30 \pm 5.00$ & \multirow{4}{*}{0.98} & \multirow{4}{*}{$0.955-0.992$} & \multirow{4}{*}{0.00} \\
\hline Tester 2 & $16.57 \pm 5.05$ & & & \\
\hline Tester 3 & $15.50 \pm 4.58$ & & & \\
\hline Tester 4 & $16.60 \pm 4.50$ & & & \\
\hline \multicolumn{5}{|c|}{ Dynamic sitting balance } \\
\hline Tester 1 & $16.57 \pm 5.94$ & \multirow{4}{*}{0.95} & \multirow{4}{*}{$0.885-0.982$} & \multirow{4}{*}{0.00} \\
\hline Tester 2 & $15.20 \pm 6.09$ & & & \\
\hline Tester 3 & $13.83 \pm 7.59$ & & & \\
\hline Tester 4 & $17.13 \pm 7.21$ & & & \\
\hline \multicolumn{5}{|c|}{ Dynamic reaching } \\
\hline Tester 1 & $8.63 \pm 1.99$ & \multirow{4}{*}{0.91} & \multirow{4}{*}{$0.811-0.967$} & \multirow{4}{*}{0.00} \\
\hline Tester 2 & $8.93 \pm 1.94$ & & & \\
\hline Tester 3 & $8.90 \pm 1.93$ & & & \\
\hline Tester 4 & $7.97 \pm 2.36$ & & & \\
\hline
\end{tabular}

translation was recommended as a means to ensure their validity by increasing the equivalence between the original and translated versions ${ }^{11)}$. In this study, translation and back translation were conducted by physiotherapy experts and specialists in English and Korean to develop the K-TCMS and facilitate the accurate semantic delivery and validation of the original content.

The objectivity and reliability of such tools are critical as they are necessary to enable accurate clinical judgments based on the scores obtained during the examination ${ }^{12}$.

Our results showed that while the K-TCMS had a high test-retest reliability, it had a relatively low inter-rater reliability. In particular, item 2, which involves lifting both arms forward, and item 12, which involves stretching the arm to reach a target, had low reliability results within 0.52 to 0.57 . This demonstrates that despite the fact that the subjects were assessed by examiners with significant clinical expertise, the scoring criteria differed among them, which influenced the reliability. Items 6 and 7, which involve bending the trunk forward or backward, require a detailed goal adjustment by considering the best trunk control range for children with $\mathrm{CP}$ as compared to normal children who can perform them more accurately. For the dynamic sitting balance test, to which functional movements were added, the qualitative aspects can be revealed clearly by children regarding whether they can or cannot perform the required motion, which allows for easier assessment. On the other hand, for motions that require bending the trunk forward or lifting the arms, which involve separation of the trunk and joints, the TCMS does not discriminate sufficiently due to a small scoring difference, which requires a fine adjustment of the scoring later. This is also applicable to items 13,14, and 15 (dynamic stretch balance), in that reaching the arms with either difficulty or ease cannot be discriminated sufficiently within a range of 0 to 2 points. For example, a 1-point difference could result in a $50 \%$ poorer performance compared to other subjects, which significantly impacts the overall assessment. Furthermore, for item 11, which involves separating and rotating the lower trunk only, all of the participating children scored 0 points because all of them had difficulties understanding and performing the motion. This item should be modified in future iterations of this tool to clarify exactly which motion is indicated by 'rotating the lower trunk'. For future studies, the above-mentioned items should be addressed or modified to increase the reliability.

The limitations of this study and implications for future studies are as follows. First, since the number of subjects was too small (15 subjects), this study result cannot be generalized to the trunk control disorders of all children with CP. Second, the subjects to whom the TCMS was applied were limited to children with spastic CP. Third, the children's age range might have been a factor that influenced the reliability. In this study, the subjects included children aged less than 8 years even though these young children not only lacked trunk control abilities, but were also still developing general postural control. In the future, vigorous studies should be conducted that take the above-mentioned issues into account, as well as a fine modification.

Despite these limitations, this study demonstrated that the K-TCMS is an assessment tool that can reliably evaluate the trunk control abilities of children with $\mathrm{CP}$. We expect that our study result will be used to further refine and standardized the $\mathrm{K}-\mathrm{TCMS}$ for use in the Korean population.

\section{ACKNOWLEDGEMENT}

This work was supported by research grants from the Daegu Catholic University in 2017. 


\section{REFERENCES}

1) Dewar R, Claus AP, Tucker K, et al.: Perspectives on postural control dysfunction to inform future research: a delphi study for children with cerebral palsy. Arch Phys Med Rehabil, 2017, 98: 463-479. [Medline] [CrossRef]

2) Kyvelidou A, Harbourne RT, Willett SL, et al.: Sitting postural control in infants with typical development, motor delay, or cerebral palsy. Pediatr Phys Ther, 2013, 25: 46-51. [Medline] [CrossRef]

3) Lee IH, Park SY: Abnormal sitting pressures of hemiplegic cerebral palsy children on a school chair. J Phys Ther Sci, 2015, 27: 499-500. [Medline] [CrossRef]

4) Saavedra S, Bellows D: Commentary on "Sitting postural control affects the development of focused attention in children with cerebral palsy". Pediatr Phys Ther, 2015, 27: 23. [Medline] [CrossRef]

5) Field D, Livingstone R: Clinical tools that measure sitting posture, seated postural control or functional abilities in children with motor impairments: a systematic review. Clin Rehabil, 2013, 27: 994-1004. [Medline] [CrossRef]

6) Bartlett D, Purdie B: Testing of the spinal alignment and range of motion measure: a discriminative measure of posture and flexibility for children with cerebral palsy. Dev Med Child Neurol, 2005, 47: 739-743. [Medline] [CrossRef]

7) Mitteregger E, Marsico P, Balzer J, et al.: Translation and construct validity of the Trunk Control Measurement Scale in children and youths with brain lesions. Res Dev Disabil, 2015, 45-46: 343-352. [Medline] [CrossRef]

8) Pham HP, Eidem A, Hansen G, et al.: Validity and responsiveness of the trunk impairment scale and trunk control measurement scale in young individuals with cerebral palsy. Phys Occup Ther Pediatr, 2016, 36: 440-452. [Medline] [CrossRef]

9) Heyrman L, Molenaers G, Desloovere K, et al.: A clinical tool to measure trunk control in children with cerebral palsy: the Trunk Control Measurement Scale. Res Dev Disabil, 2011, 32: 2624-2635. [Medline] [CrossRef]

10) Rodby-Bousquet E, Agústsson A, Jónsdóttir G, et al.: Interrater reliability and construct validity of the Posture and Postural Ability Scale in adults with cerebral palsy in supine, prone, sitting and standing positions. Clin Rehabil, 2014, 28: 82-90. [Medline] [CrossRef]

11) Sperber AD: Translation and validation of study instruments for cross-cultural research. Gastroenterology, 2004, 126: S124-S128. [Medline] [CrossRef]

12) Marsico P, Mitteregger E, Balzer J, et al.: The Trunk Control Measurement Scale: reliability and discriminative validity in children and young people with neuromotor disorders. Dev Med Child Neurol, 2017, 59: 706-712. [Medline] [CrossRef] 